\title{
Infraestructura centralizada para laboratorios de computación con escritorios virtuales
}

\author{
Centralized infrastructure for computing laboratories with virtual desks
}

Viena Muirragui Irrazábal. ${ }^{1}$, Christian Bonilla Morales. ${ }^{2}$, Edwin León Pluas. ${ }^{3}$ \& Javier Guaña Moya. ${ }^{4}$

Recibido: 22-06-2019 / Revisado: 28-07-209 /Aceptado: 09-08-2019/ Publicado: 10-09-2019

\begin{abstract}
DOI: https://doi.org/10.33262/cienciadigital.v3i3.4.836

In the present investigation a solution is designed for the universities of the city of Quito, for a virtualization solution of jobs in order to optimize both deployment and support processes, as well as reduce costs, so the technology was investigated of virtualization and the different types of virtualization that this technology currently provides, to solve different problems that exist in the data center and for end users and an analysis of the virtualization tool was carried out, which can solve the problem of universities, which is administration, access to information, without hardware purchasing processes and cost reduction.

The needs are focused on having a centralized system to deploy both virtual PCs and applications in an agile and efficient way. A design is proposed in accordance with the objectives and requirements set by the universities, dimensioning and structuring each of the elements necessary to implement the proposed solution.

Keywords: Virtualization, Desks, Laboratories, Infrastructure, Consolidation.

\section{Resumen}

En la presente investigación se diseña una solución para las universidades de la ciudad de Quito, para una solución de virtualización de puestos de trabajo con el fin de optimizar tanto procesos de despliegue y soporte, así como reducir los costos, por ello se investigó la tecnología de virtualización y los diferentes tipos de virtualización que actualmente brinda está tecnología, para solucionar diferentes problemas que se tiene en el data center y para usuarios finales y se realizó un análisis de la herramienta de virtualización, la que pueda solucionar la problemática de las universidades, que es la administración, acceso a la información, sin procesos de compras de hardware y reducción de costos.
\end{abstract}

\footnotetext{
${ }^{1}$ Universidad Estatal de Milagro, vmuirraguii1@unemi.edu.ec

${ }^{2}$ AKROS Soluciones Tecnológicas, christian.bonilla@akroscorp.com

${ }^{3}$ Universidad Estatal de Milagro, eleonp@unemi.edu.ec

${ }^{4}$ Facultad de Filosofía, Letras y Ciencias de la Educación, Universidad Central del Ecuador, ejguanam@uce.edu.ec
} 
Las necesidades se centran en disponer de un sistema centralizado para desplegar tanto PC virtuales como aplicaciones de manera ágil y eficiente. Se plantea un diseño acorde con los objetivos y requisitos planteados por las universidades, dimensionando y estructurando cada uno de los elementos necesarios para implementar la solución propuesta.

Palabras claves: Virtualización, escritorios, laboratorios, infraestructura, consolidación.

\section{INTRODUCCIÓN.}

El presente trabajo tiene el propósito de implementar de un sistema virtualizado, con el fin de brindar mayores facilidades tanto a estudiantes como docentes que utilizan los laboratorios de cómputo en las universidades del Ecuador, en donde su rendimiento se realice de manera óptima, por lo que es necesario que dicho sistema se encuentre con un diseño e implementación de manera correcta, con ello contar con ahorro de recursos en los computadores de los laboratorios, también brindar todas las comodidades necesarias para docentes y estudiantes con la utilización de herramientas tecnológicas de virtualización.

Debido al avance tecnológico que existe hoy en día, el uso de plataformas virtuales dentro del ámbito educativo ha ido evolucionando, a tal punto que se ha convertido en parte fundamental para estudiantes y docentes, es por ello que en la actualidad se cuenta con una tecnología de virtualización lo que permitirá dar mayores facilidades a los estudiantes a desarrollar las actividades de manera sencilla y optimizando recursos tecnológicos, equipamiento y espacios físicos en los laboratorios de las universidades del País.

La virtualización es una tecnología que utiliza un software específico para aprovechar al máximo las diferentes capacidades físicas de los computadores, ofreciendo un ahorro en costo y satisfaciendo las necesidades de los usuarios. La capa de virtualización (Gonzáles J. M., 2010), (Márquez, 2011), pueden ser una aplicación o directamente un sistema operativo que permite aislar los sistemas operativos virtualizados del sistema físico, proporcionándoles un hardware virtual uniforme, de este modo la memoria RAM, las CPUS, los discos duros, etc, pasan a ser recursos que se ofrecen a las máquinas virtuales para su uso (Vmware, Vmware Horizon Planificación de la Arquitectura de View, 2017). Otros beneficios incluyen el aislamiento de las máquinas virtuales y el hardware de la independencia que resulta del proceso de virtualización, las máquinas virtuales son portátiles, y se pueden mover o copiar a cualquier plataforma de hardware estándar de la industria (basado en x86), independientemente de la marca o modelo. Por lo tanto, la virtualización facilita la gestión de los recursos de TI de adaptación, y una mayor capacidad de respuesta a las cambiantes condiciones del negocio (Vmware, Vmware Horizon Guía De Usuario De Vmware Horizon cliente para Windows, 2018), (Vmware, Vmware Horizon Guía de Usuario de Vmware Horizon cliente para Linux, 2018).

Para proporcionar ventajas más allá de particionamiento, varios recursos del sistema deben ser virtualizado y administrado, incluyendo la CPU, la memoria principal, además de tener una capacidad de gestión de los recursos entre particiones. Mientras que el particionamiento es una función útil para las organizaciones de TI, la verdadera infraestructura virtual proporciona valor de negocio más allá de eso (Tapa, 2016), (Gonzáles J. M., 2011).

La plataforma de virtualización (Márquez, 2011) se construye sobre una arquitectura diseñada para el negocio, se utiliza el software para virtualizar los recursos del hardware de una computadora basada en x86 (incluidos la CPU, La memoria RAM, el disco duro y el controlador de red, y así poder crear una máquina virtual totalmente funcional, capaz 
de ejecutar su propio sistema operativo y aplicaciones como si fuera un computador físico.

La virtualización con VMware o Hyper-v introduce una capa ligera de software directamente en el Hardware del computador. Este contiene un monitor de máquina virtual o Hypervisor que asigna recursos al hardware de forma dinámica y transparente; se instalan varios sistemas operativos que funcionan simultáneamente en un solo computador y comparten recursos del hardware, gracias al encapsulamiento total de una Máquina, ya que es totalmente compatible con todos los sistemas operativos, aplicaciones y controladores de dispositivos (Curran, 2010), (Poitras, 2018), (Vmware, Administración de Vmware Virtual SAN, 2016).

El llamado Hypervisor es el núcleo central de algunas aplicaciones de virtualización este software que permite que varios sistemas operativos puedan acceder a un equipo en forma concurrente, como si cada uno de ellos fuera el dueño coordinando el acceso y uso de sus recursos, el hypervisor, permite al anfitrión compartir sus recursos de hardware a las diferentes máquinas virtuales que se generen, existen dos tipos de hypervisores, el de tipo I y el de tipo II (Castillo, 2014).

El tipo I, también conocido como nativa o bare-metal, su nombre se debe a que tiene una relación directa con el hardware del anfitrión ya que se instala directamente en el, por lo que los recursos para cada máquina virtual son administrados en su totalidad por el hypervisor (Cisco, 2016).

El tipo II, también conocido como host, no interactúa con el hardware de forma directa, sino que ahora es el sistema operativo quien dirá como administrar los recursos del equipo físico (Cisco, 2016), (Citrix, 2017).

La población que se aplicará la investigación es a 17 universidades de la ciudad de Quito, las cuales están integradas por 4 universidades públicas y 13 universidades privadas, a continuación, se nombra las Universidades de la ciudad de Quito (Galarza, 2017), (Grasso, 2006):

Del listado de las universidades se realiza un resumen que se presenta en la siguiente tabla 1

Tabla 1. Universidades de Quito

\begin{tabular}{lc}
\hline \multicolumn{1}{c}{ Tipo de Universidades } & Total \\
\hline Universidades Públicas & 4 \\
Universidades Privadas & 13 \\
Total de Universidades & 17 \\
\hline
\end{tabular}

Cada universidad tiene diferentes facultades y se tiene como resultado un promedio de 10 facultades, que utilizan las salas de cómputos. Entre las facultades que tienen más laboratorios de cómputos son las carreras de Sistemas, Informática y Electrónica,

\section{MATERIALES Y MÉTODOS.}

Se plantea la investigación basados en el análisis de soluciones de virtualización de escritorios y una posible mejora en la gestión de escritorios dentro de una red corporativa con su implementación. 
Se trazan los objetivos de la investigación que permitirán determinar la solución más adecuada para la virtualización de escritorios y comprobar si con la implementación de esta solución se mejora en la gestión de escritorios de trabajo dentro de las universidades de la ciudad de Quito.

Se justifican los motivos por los cuales se propone realizar la siguiente investigación.

Se plantea una hipótesis la cual es una posible respuesta al problema planteado y posee una íntima relación entre el problema y el objetivo.

Se realiza la recolección de datos, y se observa el comportamiento de los ambientes de pruebas con el fin de determinar cuál es la solución más adecuada para la virtualización de escritorios y posteriormente comprobar si existe una mejora en la gestión de escritorios de trabajo dentro de una red corporativa al implementar esta solución.

Se elabora las conclusiones y recomendaciones, producto del desarrollo de esta investigación.

Los índices referentes a cada indicador se evalúan cuantitativamente en varios casos y cualitativamente en otros, plasmados en tablas individuales realizadas por cada parámetro, posteriormente se elaboran tablas de resumen en donde se asignarán pesos, mediante una escala de valoración cualitativa para determinar la solución más adecuada para la virtualización de desktops (VDI).

Otro objetivo contemplado en esta investigación es determinar si existe una mejora de gestión de escritorios de trabajo al implementar una solución de virtualización de desktops (VDI).

Todos estos datos son organizados en tablas de valoración, y posteriormente aplicando métodos estadísticos se comprueba si la implementación de esta solución mejora la gestión de escritorios de trabajo, frente a la estructura tradicional de escritorios de trabajo.

Las salas de computo es poder el $100 \%$ de su tiempo a su máxima capacidad, de acuerdo con la investigación realizada, se observó que, de los 5 días hábiles de la semana, se utiliza 3 días es decir el 80\% del tiempo, (ver Figura 1)

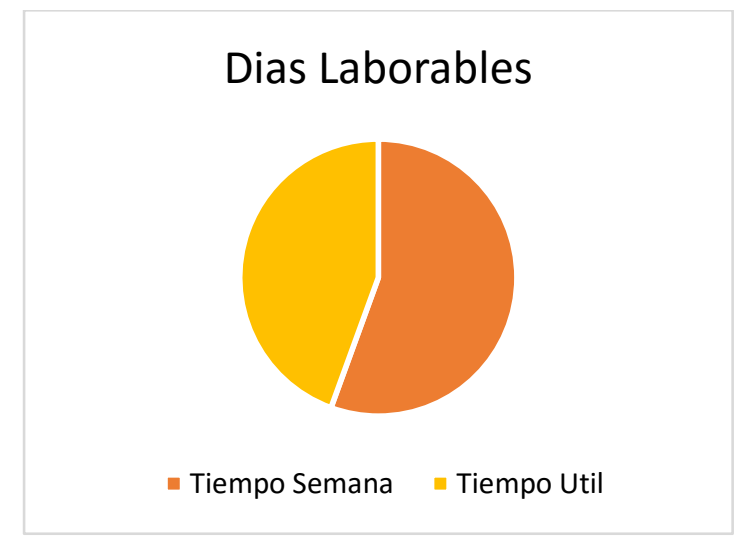

Figura 1 Tiempo útil de los laboratorios

El despliegue para que un computador puede ser utilizado por el estudiante se lleva un promedio de 1 hora por computador, este tiempo es demasiado alto cuando se tiene un laboratorio de $30 \mathrm{pc}$, ya que esto puede llevar hasta 30 horas por laboratorio, por esta razón se requiere de varias personas que puedan realizar esta actividad, si se tiene 10 
laboratorios es decir que cada semestre se necesita 300 horas para dejar hábiles las salas de computo por cada semestre y al año se requiere de 600 horas.

Los mantenimientos de los laboratorios de las universidades se realizan cada semestre, entre las principales actividades del mantenimiento es formateo de los equipos, y limpieza de los mismo, esta actividad se tiene que considerar personal externo de los administradores de los laboratorios que la mayoría son los propios estudiantes que realizan esta actividad, en las universidades se considera un grupo de 20 estudiantes para realizar los mantenimientos.

Los computadores se utilizan el $55 \%$ de su capacidad en la memoria, para que esta capacidad se aumente se debe considerar de varios aplicativos que se utilicen a la vez pero los computadores son dedicados por asignatura y su dimensionamiento se lo realiza por el aplicativo más robusto que son las bases de datos que requiere de parámetros altos para su funcionamiento, si una PC's que se utiliza las 8 horas diarias, apenas son 4,4 horas son útiles, a las semana de las 40 horas laborables que se tiene a la semana se tendrá 22 horas útiles, al año se tiene 2080 horas de las cuales 1144 horas son útiles.

Se consultó a los profesores que ellos dictan su asignatura y se utiliza los aplicativos o sistemas operativos que deben impartir en cada asignatura, existen asignaturas que no se requiere de los laboratorios de cómputos, pero tienen la obligación de utilizarlo, es decir los laboratorios también se deben utilizar para realizar consultas de temas relacionados con la asignatura, considerando esto que los PC's no son utilizados a su máxima capacidad.

Los estudiantes en la entrevista que se realizó, ellos indicaron que el uso del laboratorio se tiene acceso en los horarios establecidos a la asignatura, después de ese horario no se tiene acceso a la información del laboratorio, y esto perjudica al aprendizaje de cada estudiante, en este trabajo investigativo debe dar una solución a este problema.

Actualmente los laboratorios cuentan con una administración compleja, las universidades contemplan un administrador por cada 2 laboratorio, es decir si las universidades tienen 10 laboratorio estos son administrados por 5 personas, y existe un administrador general que lleva el control sobre los administradores de los laboratorios, en la Figura 2 se muestra una arquitectura de los laboratorios actualmente.

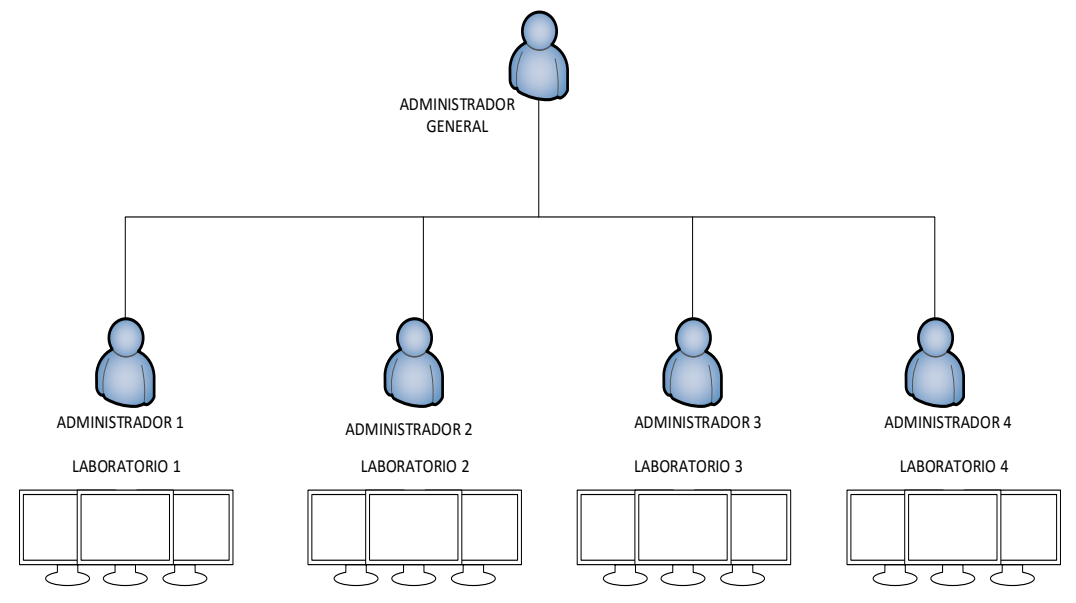

Figura 2 Arquitectura de Laboratorios 
Para este análisis, se evaluaron los softwares de virtualización de escritorios y aplicaciones y se estableció el alcance y características generales que estos deben brindar y cubrir las necesidades de las universidades, se elaboró un cuadro comparativo de las principales características que debe de contar el software de virtualización de escritorio y aplicaciones el cual se describe a continuación

Tabla 2. Cuadro comparativo

\begin{tabular}{|c|c|c|c|}
\hline Item & Características Técnicas & $\begin{array}{l}\text { Citrix } \\
\text { XenDesktop y } \\
\text { Xenapp }\end{array}$ & VMWare Horizon \\
\hline 1 & $\begin{array}{l}\text { Compatibilidad con S.O. Windows } 10 \text {, } \\
\text { Windows } 7 \text {, Windows Server, SO } \\
\text { Linux Ubuntu, Centos, NeoKylin y IOS }\end{array}$ & SI & SI \\
\hline 2 & $\begin{array}{l}\text { Acceso a escritorios virtuales y } \\
\text { aplicaciones Virtuales }\end{array}$ & SI & SI \\
\hline 3 & $\begin{array}{l}\text { Acceso al escritorio y aplicación virtual } \\
\text { a través de un explorador web }\end{array}$ & SI & SI \\
\hline 4 & Integración con Active Directory & SI & SI \\
\hline 5 & $\begin{array}{l}\text { Aprovisionamiento de los escritorios } \\
\text { virtuales }\end{array}$ & $\begin{array}{l}5 \text { (Provisioning } \\
\text { server) }\end{array}$ & $\begin{array}{c}4 \\
\text { (View Composer) }\end{array}$ \\
\hline 6 & Acceso con HTML5 & SI & SI \\
\hline 7 & $\begin{array}{l}\text { Acceso a los escritorios virtuales y } \\
\text { aplicativos desde Internet (publicados) }\end{array}$ & $\begin{array}{l}\text { SI, Nativo } \\
\text { Netscaler }\end{array}$ & $\begin{array}{l}\text { SI, a través de } \\
\text { terceros } \\
\text { F5 }\end{array}$ \\
\hline 8 & $\begin{array}{l}\text { Acceso seguro SSL a las aplicaciones y } \\
\text { escritorios virtualizados desde fuera de } \\
\text { la organización }\end{array}$ & SI & SI \\
\hline 9 & $\begin{array}{l}\text { Eficiencia en la transmisión (consumo } \\
\text { de ancho de banda) de los escritorios y } \\
\text { aplicaciones virtuales }\end{array}$ & $\begin{array}{l}\text { SI, Protocolo } \\
\text { ICA }\end{array}$ & SI, Protocolo PCoIP \\
\hline 10 & $\begin{array}{l}\text { Administración y Gestión de imágenes } \\
\text { centralizada (incorporación de perfiles } \\
\text { de usuarios para escritorios y } \\
\text { servidores). }\end{array}$ & $\begin{array}{l}\text { SI, Consolas } \\
\text { administrativas } \\
\text { XenApp y } \\
\text { XenDesktop }\end{array}$ & $\begin{array}{l}\text { SI, VMWare Mirage, } \\
\text { una sola consola para } \\
\text { administrar todos sus } \\
\text { productos }\end{array}$ \\
\hline 11 & $\begin{array}{l}\text { Capacidad para alojar y publicar } \\
\text { cualquier aplicación (16, } 32 \text { y } 64 \text { bit / } \\
\text { incompatible con SO de servidor) } \\
\text { mediante aplicaciones alojadas en VM }\end{array}$ & SI & SI \\
\hline 12 & $\begin{array}{l}\text { Amplia compatibilidad con periféricos } \\
\text { (como USB, COM, LPT, webcams, } \\
\text { micrófonos, unidades cliente, etc.) }\end{array}$ & $\begin{array}{l}\text { SI, Mediante } \\
\text { equipos } \\
\text { Zero Client }\end{array}$ & $\begin{array}{l}\text { SI, Mediante equipos } \\
\text { Zero Client }\end{array}$ \\
\hline
\end{tabular}

La herramienta de virtualización que se analizará es Vmware Horizon View que transforma los escritorios estáticos en espacios de trabajo digitales y seguros que se puedan entregar bajo demanda, aprovisionar escritorios y aplicaciones virtuales por medio de una plataforma única de virtualización de aplicaciones y de infraestructura de escritorio virtual (Virtual Desktop Infrastructure, VDI). 
Distribuye los escritorios virtuales o remotos y aplicaciones a través de una única plataforma de VDI a fin de simplificar la gestión y facilitar la asignación de derechos a los usuarios finales.

Proporciona una experiencia de usuario final óptima a trabajadores finales, trabajadores móviles e incluso desarrolladores de 3D desde cualquier dispositivo, ubicación, soporte y conexión BYOD (Brig your own Device) como IOS, Android, Windows, MAC OSX y Linux.

Consolida el control, el despliegue y la protección de los recursos informáticos del usuario mediante la gestión y automatización integrales. Aprovecha las redes virtuales a fin de definir políticas de seguridad de forma sencilla y dinámica para grupos de usuarios finales.

Se puede asignar recursos de forma dinámica gracias al almacenamiento virtual, los entornos informáticos virtuales y las redes virtuales a fin de simplificar la gestión y reducir los costos.

Al virtualizar los puestos de trabajo se reduce los costos operativos diarios ostensiblemente con una única plataforma que permite extender la virtualización desde el centro de datos a los dispositivos, permite a las organizaciones ampliar la potencia de la virtualización de escritorios y aplicaciones para respaldar la movilidad del espacio de trabajo, además de ofrecer mayores niveles de eficiencia operativa con un costo inferior.

\section{RESULTADOS Y DISCUSION.}

El aplicativo "Ireport" requiere de una memoria de $8 \mathrm{~GB}$, procesador de $2,5 \mathrm{GHz}$ y un almacenamiento de 40GB son los requerimientos mínimos que necesita para su instalación, por esta razón el perfil de los computadores que se tiene en los laboratorios de computo tienen las siguientes características.

- Procesador: Core i7 de 3,60 GHz

- Memoria instalada: (RAM) 8 GB

- Discos: $1 \mathrm{~Tb}$

Actualmente los equipos de cómputos vienen con características muy altas que son accesibles a las entidades educativas y que permiten tener memoria robusta, procesadores rápidos y almacenamiento de gran capacidad.

Para determinar el rendimiento de los equipos se realizó tomas de las estadísticas del uso de la memoria y delo procesador que son los recursos más utilizados en una PC, la toma se realizó en el momento que se ejecutaban 7 herramientas. Entre los aplicativos que se ejecutó esta Ireport que los estudiantes utilizan para realizar las actividades en la asignatura, en la figura 3 se observa que el recurso más utilizado es la memoria con el $55 \%$ y su procesador al $80 \%$ 


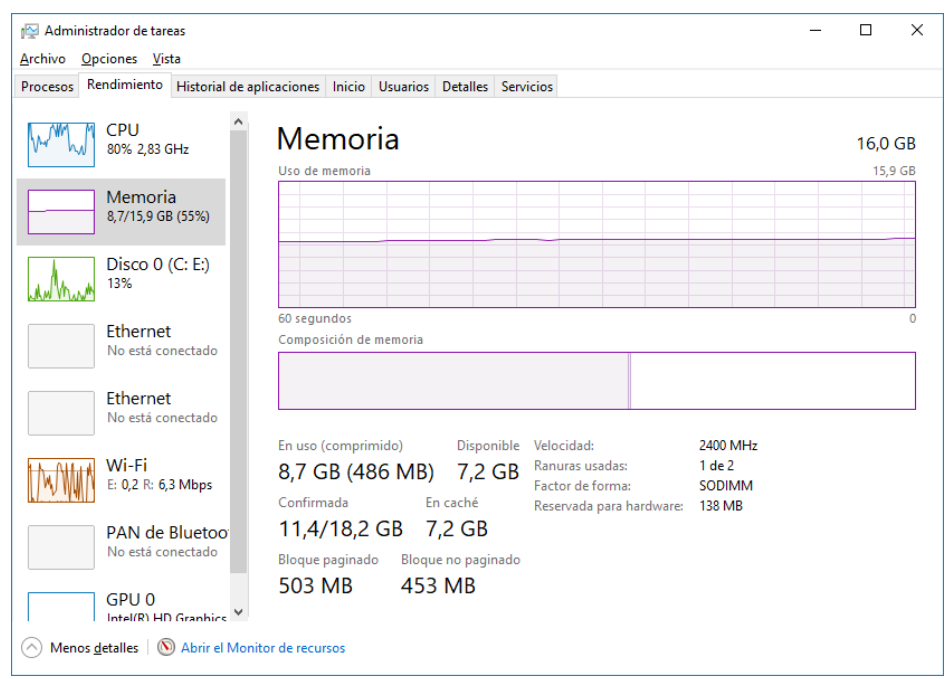

Figura 3. Rendimiento de PC

En el proyecto de virtualización de escritorio el recurso más importante es la memoria, el rendimiento del 55\% de su capacidad, se debe mejorar su capacidad al $85 \%$, esto es viable por la tecnología de los escritorios virtuales mejorando considerablemente su rendimiento por computador.

\subsection{Requisitos de hardware para el servidor de conexión de Horizon}

Todos los tipos de instalaciones del servidor de conexión de Horizon, incluidas las instalaciones estándar, réplica, del servidor de seguridad y del servidor de inscripciones, en una máquina virtual o en un equipo físico dedicados que cumplan los requisitos específicos de hardware.

Tabla 4. Requisitos de hardware del servidor de conexión de Horizon

\begin{tabular}{lll}
\multicolumn{1}{c}{$\begin{array}{c}\text { Componente de } \\
\text { hardware }\end{array}$} & \multicolumn{1}{c}{ Obligatorio } & \multicolumn{1}{c}{ Recomendado } \\
\hline Procesador & $\begin{array}{l}\text { Procesador Pentium IV } \\
\text { de 2 GHz o superior }\end{array}$ & 4 CPU \\
Adaptador de red & NIC de 100 Mbps & NIC de 1 Gbps \\
Memoria & 4 GB de RAM o superior & $\begin{array}{l}\text { Al menos 10 GB de RAM o } \\
\text { superior para implementaciones } \\
\text { de 50 o más escritorios remotos }\end{array}$ \\
$\begin{array}{l}\text { Windows Server 2008 } \\
\text { R2 de 64 bits }\end{array}$ & Al menos 10 GB de RAM o \\
Memoria & 4 GB de RAM o superior & $\begin{array}{l}\text { superior para implementaciones } \\
\text { de 50 o más escritorios remotos }\end{array}$ \\
Windows Server 2012 & & \\
R2 de 64 bits & &
\end{tabular}

Tabla 5. Sistemas operativos compatibles con el servidor de conexión de Horizon

\begin{tabular}{lcc}
\hline \multicolumn{1}{c}{ Sistema Operativo } & Versión & Edición \\
\hline Windows Server 2008 R2 & 64 bits & Standard \\
SP1 & & Enterprise
\end{tabular}




\begin{tabular}{lcc} 
& & Data Center \\
Windows Server 2012 R2 & 64 bits & Standard \\
& & Data Center \\
Standard \\
Windows Server 2016 & 64 bits & Data Center \\
\hline
\end{tabular}

Al instalar instancias replicadas del servidor de conexión de Horizon, se configura en la misma ubicación física y conectarlas a una red LAN de alto rendimiento. Caso contrario, los problemas de latencia podrían provocar inconsistencias en las configuraciones LDAP de View de las instancias del servidor de conexión de Horizon. Si la configuración de una instancia del servidor de conexión de Horizon no está actualizada, es posible que se rechace el acceso del usuario cuando este se conecta.

\subsection{Requisitos de hardware y base de datos para un View Composer independiente}

El componente de View Composer puede ser una máquina virtual o en un equipo físico diferentes de la usada para vCenter Server, debe usar una máquina dedicada que cumpla los requisitos de hardware específicos. Una instalación de View Composer independiente funciona con vCenter Server instalado en un equipo Windows Server o con el dispositivo de vCenter Server basado en Linux. VMware recomienda tener una asignación uno a uno entre el servicio View Composer y la instancia de vCenter Server.

Tabla 6. Requisitos de hardware para View Composer

\begin{tabular}{lll}
\multicolumn{1}{c}{$\begin{array}{c}\text { Componente de } \\
\text { hardware }\end{array}$} & \multicolumn{1}{c}{ Obligatorio } & \multicolumn{1}{c}{ Recomendado } \\
\hline Procesador & $\begin{array}{l}\text { Intel 64 de 1,4 GHz o más } \\
\text { potente, o bien procesador AMD 64 } \\
\text { con 2 CPU } \\
\text { Una o varias tarjetas de interfaz de } \\
\text { red (NIC) de 10/100 Mbps }\end{array}$ & $\begin{array}{l}2 \mathrm{GHz} \text { o más potente y } 4 \\
\mathrm{CPU}\end{array}$ \\
& NIC de 1 Gbps \\
Red & 4 GB de RAM o superior & $\begin{array}{l}\text { 8 GB de RAM o superior } \\
\text { para implementaciones de } \\
50\end{array}$ \\
Memoria & o más escritorios remotos \\
& 40GB & 60GB \\
\hline
\end{tabular}

View Composer necesita una base de datos SQL para almacenar datos. La base de datos debe residir o estar disponible en el host del servidor de View Composer. Puede configurar de forma opcional una base de datos de eventos para registrar información desde el servidor de conexión de View sobre los eventos de View.

Si una instancia del servidor de la base de datos ya existe para vCenter Server, View Composer puede usar esa instancia existente si es una de las versiones que aparece en Tabla 1-5. Por ejemplo, View Composer puede usar la instancia de Microsoft SQL Server proporcionada con vCenter Server. Si no existe ninguna instancia del servidor de la base de datos, debe instalar una. View Composer admite un subconjunto de servidores de la base de datos que vCenter Server admite.

Si ya utiliza vCenter Server con un servidor de la base de datos que View Composer no admita, continúe usando el servidor de la base de datos para vCenter Server e instale un servidor de la base de datos independiente para usar View Composer. 
Tabla 7. Requisitos base de datos para View Composer

\begin{tabular}{lcc}
\hline \multicolumn{1}{c}{ Base de datos } & $\begin{array}{c}\text { Versiones/paquetes } \\
\text { de servicio }\end{array}$ & Ediciones \\
\hline $\begin{array}{l}\text { Microsoft SQL Server 2014 } \\
\text { (32 y 64 bits) }\end{array}$ & Sin SP, SP1 & Standard \\
Eicrosoft SQL Server 2012 & SP2 & Express \\
(32 y 64 bits) & & Standard \\
Microsoft SQL Server 2008 & SP2, SP3 & Express \\
R2 & & Standard \\
(32 y 64 bits) & Enterprise \\
& Versión 1 (cualquier & Datacenter \\
& versión hasta & Standard One \\
Oracle 12c & $12.1 .0 .2)$ & Standard \\
& & Enterprise \\
\hline
\end{tabular}

\subsection{Fase 2 Diseño y Despliegue del ambiente virtual}

Para el diseño del ambiente virtual que se requiere de hardware que se utilizara en el data center donde estarán alojados los escritorios virtuales y se requieres de la herramienta de virtualización que brindará los beneficios de los escritorios virtuales.

\section{Arquitectura Diseño}

Para el trabajo de investigación, el diseño se basó en 300 escritorios que las universidades tienen actualmente en los laboratorios que están distribuidos en las diferentes asignaturas, en la Figura 4 se muestra el diagrama lógico de la arquitectura propuesta.

La arquitectura está compuesta por

- servidores los cuales están en cluster virtualizados

- $\quad$ software de virtualización de servidores

- software de virtualización de escritorios virtuales

- switch de core a 10GB

- balanceadores de carga para el acceso desde el internet

La administración se lo realizará desde una sola consola la cual estará a cargo de una persona reduciendo el personal considerablemente en la administración.

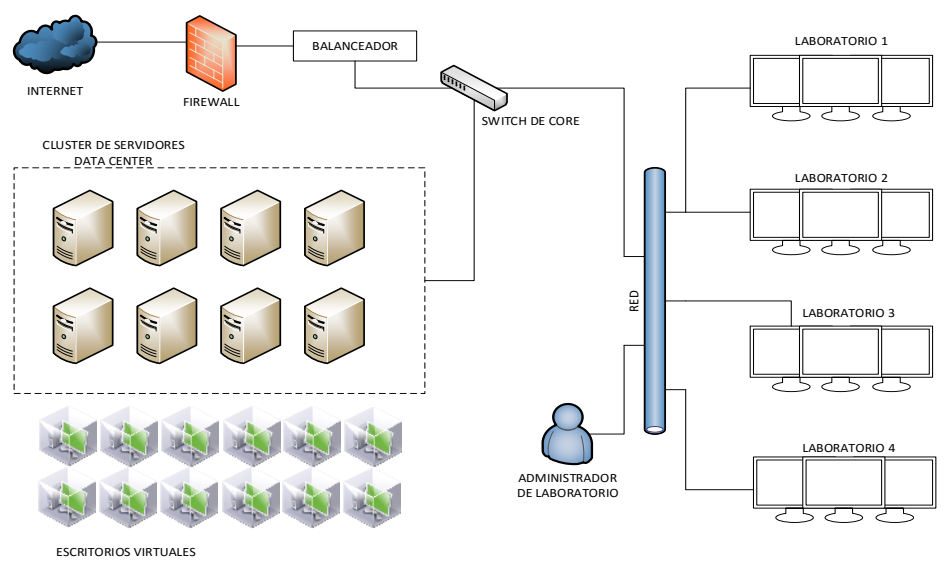

Figura 4. Arquitectura Lógica Propuesta 
En la figura 5 se muestra la arquitectura física que se propone a las universidades de la ciudad de Quito (Bonilla Morales, 2019).

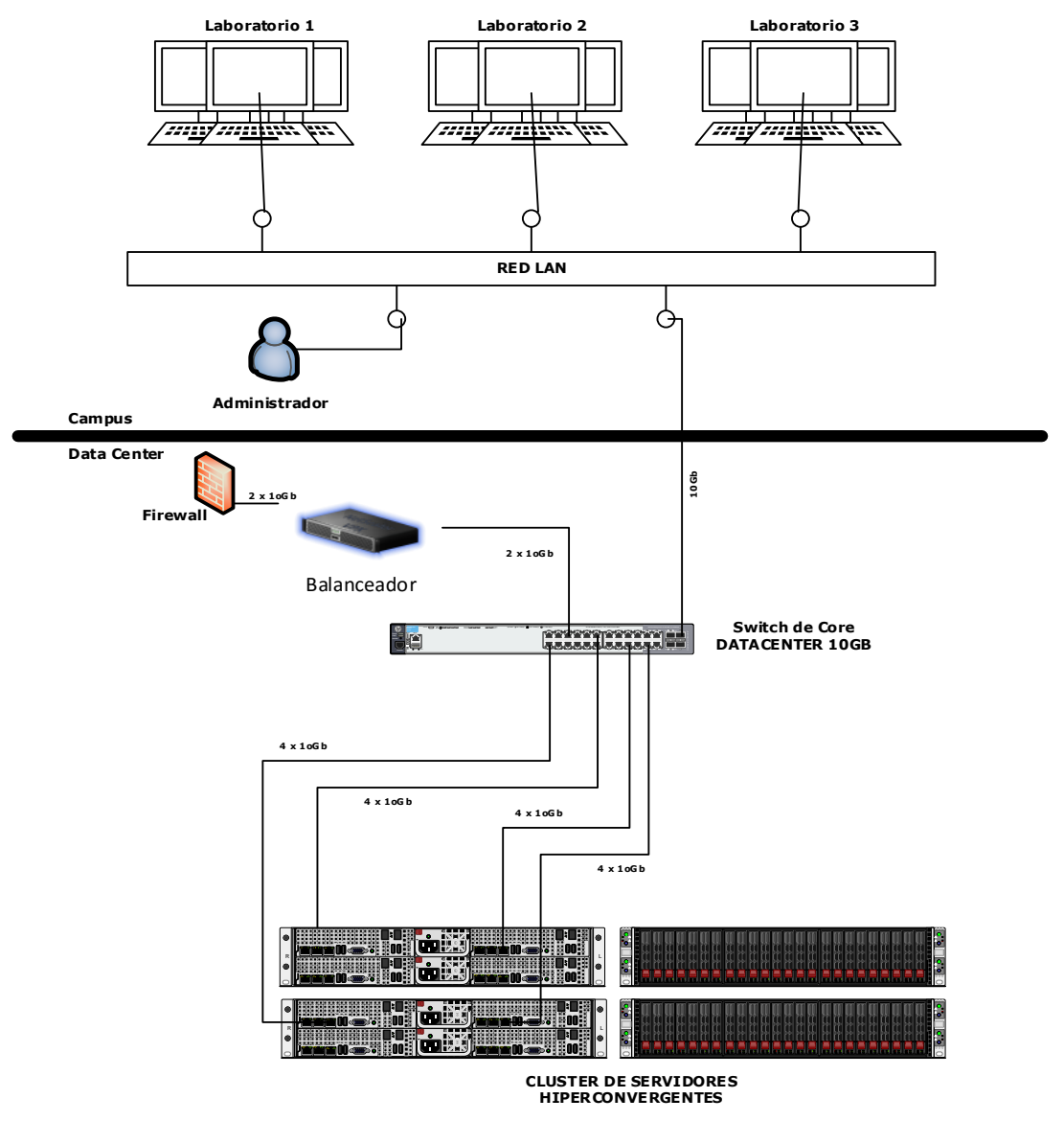

\section{Figura 5 Arquitectura Física Propuesta}

Para el diseño de la propuesta para las universidades de la ciudad de Quito, se realiza para 300 escritorios virtuales de acuerdo al análisis en la cual se determinó que se tiene un promedio de 10 laboratorios por las facultades y que cada laboratorio tiene 30 computadores; para el diseño ha considerado un perfil, los parámetros principales que se consideran para el desarrollo del diseño son los siguientes:

\section{Perfil Único:}

- $\quad$ Procesador: $1 \mathrm{Vcpu}$

- Memoria: $6 \mathrm{~Gb}$ esto se determinó de que la memoria que tienen actualmente los equipos físicos es recurso que no se lo está aprovechando al 100\% por esta razón se determinó bajar su capacidad

- Almacenamiento o Disco: Se ha considerado 60GB, se debe recalcar que este espacio se encuentra en le data center principal y solo es para guardar la información que el estudiante genere en el laboratorio. 
Tabla 8. Perfil de PC's

\begin{tabular}{lcl}
\hline \multicolumn{1}{c}{ PARAMETRO } & CANTIDAD & \multicolumn{1}{c}{ OBSERVACIONES } \\
\hline USUARIOS & 300 & Se necesita 1 procesador por escritorio virtual \\
PROCESADOR & 1 & $\begin{array}{l}\text { Memoria se requiere por los aplicativos de } \\
\text { cada asignatura }\end{array}$ \\
MEMORIA (GB) & 6 & $\begin{array}{l}\text { Este parámetro es solo para los datos que } \\
\text { genere el estudiante por SO y APP están } \\
\text { instalados en el data center principal }\end{array}$ \\
$\begin{array}{l}\text { ALMACENAMIENTO } \\
\text { (GB) }\end{array}$ & 60 & \\
\hline
\end{tabular}

Para el dimensionamiento del equipamiento para el Data Center se determina por los recursos principales que son los más utilizados en el dimensionamiento de los escritorios virtuales

Tabla 9. Recursos de Data Center

\begin{tabular}{lcl}
\hline $\begin{array}{l}\text { RECURSOS DEL } \\
\text { DATA CENTER }\end{array}$ & CAPACIDAD & \multicolumn{1}{c}{ DESCRIPCION } \\
\hline $\begin{array}{l}\text { ALMACENAMIENTO } \\
\text { MEMORIA }\end{array}$ & 18000 & $\begin{array}{l}\text { Es la capacidad de almacenamiento mínima que debe } \\
\text { tener para cubrir todos los escritorios virtuales } \\
\text { Capacidad de memoria requerida para cubrir la } \\
\text { totalidad de los escritorios virtuales }\end{array}$ \\
$\begin{array}{l}\text { SERVIDORES } \\
\text { VROCESADORES }\end{array}$ & 2400 & El cluster de servidores debe brindar 300 Vcpu \\
\hline
\end{tabular}

Para el dimensionamiento del procesador de los escritorios virtuales que se requiere:

Tabla 10. Dimensionamiento Procesamiento

\begin{tabular}{lcl}
\hline $\begin{array}{c}\text { RECURSOS DEL DATA } \\
\text { CENTER }\end{array}$ & CAPACIDAD & \multicolumn{1}{c}{ DESCRIPCION } \\
\hline $\begin{array}{l}\text { TIPO DE } \\
\text { PROCESADOR }\end{array}$ & Intel Skylake 6140 & $\begin{array}{l}\text { Procesadores actuales y que su desempeño } \\
\text { es para virtualización }\end{array}$ \\
$\begin{array}{l}\text { CORE POR } \\
\text { PROCESADOR }\end{array}$ & 18 & $\begin{array}{l}\text { Cada procesador tiene } 18 \text { core } \\
\text { NUMERO DE }\end{array}$ \\
PROCESADORES & 8 & $\begin{array}{l}\text { El número de servidores requeridos es de } 4 \\
\text { servidores o nodos de computo por el } \\
\text { factor de protección }\end{array}$ \\
TOTAL DE CORE & 144 & $\begin{array}{l}\text { Por el cluster de servidores o nodos } \\
\text { La conversión que se tendrá es por } 1 \text { core } \\
\text { físico se tendrá } 4 \text { vcpu }\end{array}$ \\
\hline
\end{tabular}

Para el diseño del hardware se considera 4 equipos de cómputo por el factor de protección que sebe tener para la solución de los equipos, cada servidor o nodo de computo debe tener 2 procesadores de acuerdo al dimensionamiento que se realizó.

Cada servidor o nodo de computo debe tener mínimo 600GB de memoria, actualmente los servidores cuentas o 24 slots para memoria y se recomienda que los dimm de memoria debe ser de 64GB, pero por balanceo de carga de memoria se debe llegar a una capacidad de 768G (Ver figura 6). 
El almacenamiento se debe considerar un porcentaje de discos de estado sólido porque esta tecnología de disco permite tener un rendimiento de IOPS en las máquinas virtuales, permitiendo que la experiencia del usuario final sea óptima.

Para el diseño se recomienda una solución de hiper convergencia porque se tiene una actualización de la tecnología en el data center con 4 nodos de cómputos en solo $4 \mathrm{U}$ de rack teniendo un ahorro considerable en el espacio de rack, aire acondicionado y consumo eléctrico, permitiendo a la Universidades el ahorro en estos rubros.
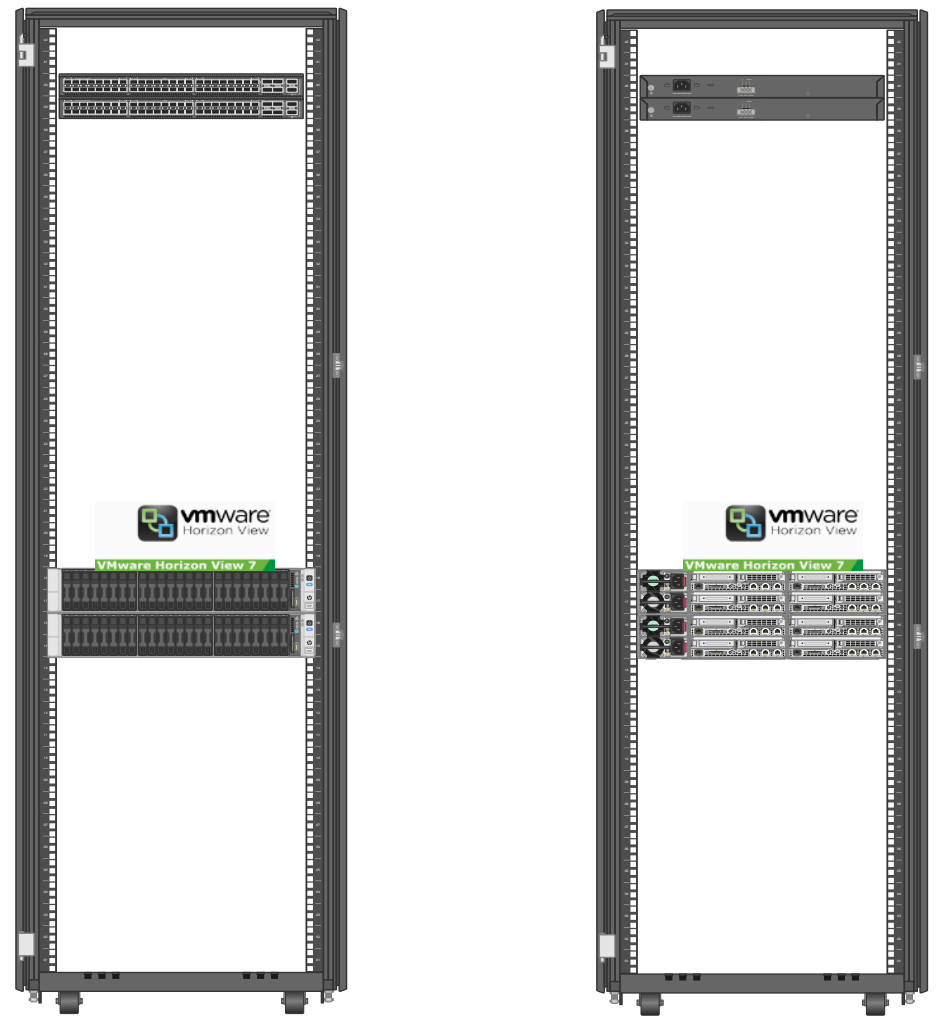

\section{Figura 6 Arquitectura Fisica Hiperconvergente}

Se realizó un prototipo el cual se instaló la herramienta de Horizon View en un servidor y se desplego 10 escritorios virtuales, en los cuales se instaló sistema operativo tanto windows y Linux, y se instaló los aplicativos que se tiene actualmente en los laboratorios.

\section{CONCLUSIONES.}

Al realizar el análisis comparativo de las dos soluciones de virtualización se verificó que la herramienta que cubre las necesidades de las Universidades es Horizon View, esta herramienta permite tener un rendimiento en la solución de VDI, administración, seguridad, flexibilidad en la implementación, acceso a un portal de servicios y gestión de una solución de escritorios de trabajo.

Con la virtualización de escritorios se mejorar el tiempo de habilitar un escritorio virtual para ser usado por el estudiante, el tiempo estimado para habilitar 300 escritorios 
virtuales es de 32 horas que representa el $25 \%$ del tiempo que actualmente se empleando para tener habilitado los laboratorios en las universidades.

El consumo eléctrico es otro parámetro fundamental en el ahorro para el trabajo de investigación, con la virtualización de escritorios, se emplea menos servidores que tienen un consumo eléctrico mínimo, especio físico en el rack y menos aire acondicionado, con respecto a las PC el ahorro del consumo eléctrico llega hasta un $90 \%$ de ahorro, esto a las entidades educativo es un impacto en las planillas eléctricas.

Se dispone de la información a su $100 \%$ con la alta disponibilidad en hardware y el software se tiene la información las 24 horas del día, los 7 días de la semana y los 365 días del año incluido días feriados, la disponibilidad se tiene desde cualquier parte del país y desde el cualquier dispositivo, esto no pasa actualmente por la tecnología que se tiene en los laboratorios.

Dentro de las soluciones de virtualización de desktops se mejora los procesos de seguridad de los datos frente a la infraestructura tradicional, Horizont View permite definir políticas de seguridad a nivel de grupos no a nivel de usuarios, usando un cifrado para evitar que usuarios no autorizado pueda acceder al escritorio de trabajo ya que usan Active Directory dentro de su configuración.

\section{REFERENCIAS}

Bonilla Morales, C. L. (2019). Optimización de la Infraestructura de Tecnologías de la Información Utilizando Escritorios Virtuales. Bachelor's thesis, Quito.

Castillo, E. (2014). Sistemas Operativos.

Cisco, A. (2016). Cisco Data Center Spine and Leaf Architecture: Design Overview.

Citrix, S. (2017). Xenapp y Xendesktop 7.14 .

Curran, C. (2010). Fedora 13 Manual de Virtualización La Guía Definitiva de Virtualización En Fedora Edición.

Galarza, K. (2017). Tecnología de Virtualización Reducción de Costos y Múltiples Beneficios con Vmware .

Gonzáles, J. M. (2010). Secretos de Vmware. Vsphere - Segunda Edición.

Gonzáles, J. M. (2011). Descubre y Domina Vmware Vsphere .

Grasso, L. (2006). Elementos para su diseño y análisis. Córdoba, Argentina.

Márquez, A. (2011). irtualización de Servidores.

Poitras, S. (2018). The Nutanix Bible.

Tapa, B. (2016). Implementing vmware horizon 7. 
Vmware, I. (2016). Administración de Vmware Virtual SAN.

Vmware, I. (2017). Vmware Horizon Planificación de la Arquitectura de View.

Vmware, I. (2018). Vmware Horizon Guía de Usuario de Vmware Horizon cliente para Linux.

Vmware, I. (2018). Vmware Horizon Guía De Usuario De Vmware Horizon cliente para Windows. 


\section{PARA CITAR EL ARTÍCULO INDEXADO.}

Muirragui Irrazábal, V., Bonilla Morales, C., León Pluas, E., \& Guaña Moya, J. (2019). Infraestructura centralizada para laboratorios de computación con escritorios virtuales. Ciencia Digital, 3(3.4.), 75-90.

https://doi.org/10.33262/cienciadigital.v3i3.4.836

\section{Liencia}

El artículo que se publica es de exclusiva responsabilidad de los autores y no necesariamente reflejan el pensamiento de la Revista Ciencia Digital.

El artículo queda en propiedad de la revista y, por tanto, su publicación parcial y/o total en otro medio tiene que ser autorizado por el director de la Revista Ciencia Digital.
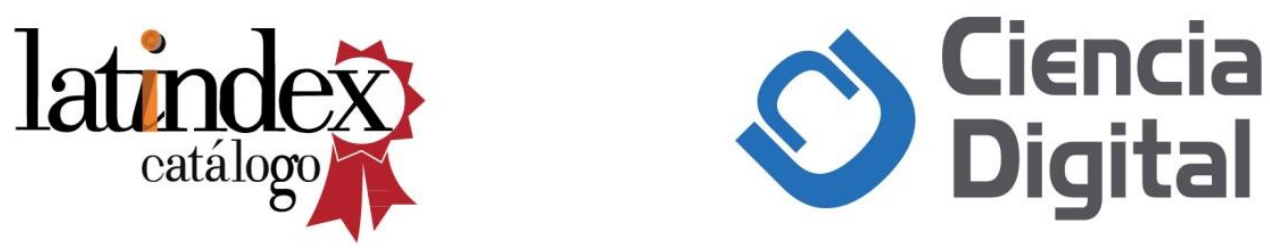